\title{
Neuroblastoma olfatorio. Reporte de
}

\section{caso}

\author{
DOI 10.5377/alerta.v5i1.12817 \\ Ronald Josué Hernández Prudencio ${ }^{1 *}$, José Alvarino Minero Ortiz ${ }^{2}$ \\ 1-2. Hospital General del Instituto Salvadoreño del Seguro Social, San Salvador, El Salvador \\ ${ }^{*}$ Correspondencia \\ $\square$ ronaldj_2060@hotmail.com \\ 1. (1) 0000-0003-4116-3802
}

\section{f ACCESO ABIERTO}

Olfactory neuroblastoma. Case report

\section{Citación recomendada:}

Hérnandez Prudencio

RJ, Minero Ortiz JA.

Neuroblastoma olfatorio.

Reporte de caso. Alerta.

2022;5(1):12-16. DOI: 10.5377/

alerta.v5i1.12817

\section{Recibido:}

1 de septiembre de 2021.

\section{Aceptado:}

25 de septiembre de 2021.

\section{Publicado:}

27 de enero de 2022

\section{Contribución de autoría: \\ RJHP': recolección de datos, análisis del caso, redacción, revisión, edición y búsqueda bibliográfica. JAMO²: concepción del estudio, diseño del manuscrito y análisis del caso}

\section{Conflicto de intereses:}

Los autores declaran no tener ningún tipo de conflicto de interés.

\begin{abstract}
Resumen
Presentación del caso. Hombre de 36 años, con cuadro de inflamación palpebral derecha de tres meses de evolución, acompañado de epífora, fotofobia y en el ojo derecho únicamente presentaba percepción de estímulos luminosos. Los estudios radiológicos identificaron cambios en la densidad de los senos paranasales a predominio derecho y ocupación a nivel del seno maxilar derecho. Intervención terapéutica. Se realiza exéresis quirúrgica completa de la lesión. De acuerdo con los hallazgos inmunohistoquímicos se diagnosticó un neuroblastoma olfatorio de la región nasal derecha. Debido a la severidad y la rápida progresión de la enfermedad. Se incluyeron cuidados neurocríticos posteriores a la intervención, ventilación mecánica con traqueostomía y soporte aminérgico. Evolución clínica. Su evolución no fue favorable; el paciente falleció después de 18 días de estancia hospitalaria.

\section{Palabras clave}

Neuroblastoma olfatorio, epitelio olfatorio, cavidad nasal
\end{abstract}

\begin{abstract}
Case presentation. A 36-year-old man, with a three-month history of right palpebral swelling, accompanied by epiphora, photophobia and in the right eye, he only presented perception of light stimulus. Radiological studies identified changes in the density of the paranasal sinuses, mainly on the right, and occupation at the level of the right maxillary sinus. Treatment. Complete surgical excision of the lesion was required. According to the immunohistochemical findings, an olfactory neuroblastoma of the right nasal region was diagnosed. Due to the severity and rapid progression of the disease, were included post-operative neurocritical care, mechanical ventilation with tracheostomy and aminergic support. Outcome. Evolution was unfavorable, the patient died after 18 days of hospital stay.

Keywords

Olfactory neuroblastoma, neuroepithelioma, olfactory mucosa.
\end{abstract}

\section{Introducción}

El neuroblastoma olfativo, también conocido como estesioneuroblastoma', es un tumor derivado de la capa basal del epitelio olfativo 2 . Se presenta como una masa de tejido blando en la porción superior de la cavidad nasal que involucra las células de aire etmoides anteriores y medias en un lado y se extiende a través de la placa cribiforme en la fosa craneal anterior ${ }^{1,2}$.

En 1924, Berger, Luc y Richard describieron un tumor nervioso olfativo que se parecía a un retinoblastoma histológicamente, llamándolo neuroblastoma olfatorio.
Dos años más tarde, Berger y Coutard ${ }^{4}$, describieron un segundo tumor neurogénico intranasal que difiere un poco en el patrón histológico y lo denominan estesioneurocitoma. Seaman y Schall y Lineback ${ }^{5}$ reportaron por primera vez este tumor en la literatura estadounidense en 1951.

El neuroblastoma olfatorio es poco frecuente y representa menos del $3 \%$ de las neoplasias intranasales, ${ }^{1,2}$. De acuerdo con la evolución epidemiológica, su distribución por edades es bimodal con un pico en pacientes adultos en la segunda década de la vida y otro en los quinta y sexta, sin existir predilección por género reconocida ${ }^{1,6}$. 


\section{Presentación del caso}

Hombre de 36 años, originario de la zona central de El Salvador, sin antecedentes médicos, quirúrgicos o familiares relevantes, quien consultó en el Hospital General del Instituto Salvadoreño del Seguro Social en abril de 2020, debido a un cuadro de inflamación palpebral derecha, acompañado de epífora y fotofobia en el ojo derecho de 3 meses de evolución con manejo de aparente conjuntivitis alérgica en una unidad periférica, sin presentar mejoría clínica.

En el examen físico se encontró afebril, alerta, orientado, con frecuencia cardíaca de 66 latidos por minuto, frecuencia respiratoria de 14 por minuto y presión arterial de 120/70 mmHg. En la exploración craneofacial se identificó el edema palpebral derecho con dolor leve a la palpación, acompañado de rubor, calor y en el ojo derecho presentaba únicamente percepción de estímulos luminosos. En la rinoscopía se observó un tumor de aspecto polipoideo que ocupaba el $70 \%$ de la fosa nasal derecha. Estaba ubicado en el tercio superior de la fosa nasal y se extendía hacia el septum nasal y al cornete medio sin invadirlos; los cornetes inferiores no tenían infiltración. La fosa nasal izquierda presentó lesiones de tipo tumoral o inflamatoria. Sin otra anormalidad al examen físico.

Los datos de laboratorio indicaron anemia leve (hemoglobina de 11,6 g/dl), sin leucocitosis, ni neutrofilia, las plaquetas, creatinina y glucosa al azar se encontraban en los rangos normales y proteína $C$ reactiva de 1,22 mg/L. La radiografía de senos paranasales, en proyecciones lateral y derecha Cadwell, describió asimetría con pérdida de la diferenciación de los cornetes nasales a predominio derecho y asimetría de los tejidos blandos de región frontonasal (Figura 1).

Se realizaron otros estudios imagenológicos de los senos paranasales. La tomografía axial computarizada describió una masa de tejidos blandos con infiltración en ambos senos maxilares, paredes mediales de las órbitas y desplazamiento al músculo recto medial derecho lateralmente. La resonancia magnética cerebral identificó una lesión de características infiltrantes a lámina cribiforme y hueso frontal con extensión a región intraconal derecha. Se clasificó según Kadish como tipo C1 (Figura 2).

Los hallazgos imagenológicos fueron evaluados por otorrinolaringología y neurocirugía, quienes analizaron la progresión rápida y el estadio de la tumoración nasal, compatible con un neuroblastoma olfatorio, con diagnósticos probables de un rabdominosarcoma, un carcinoma nasofaríngeo o un carcinoma sinonasal.
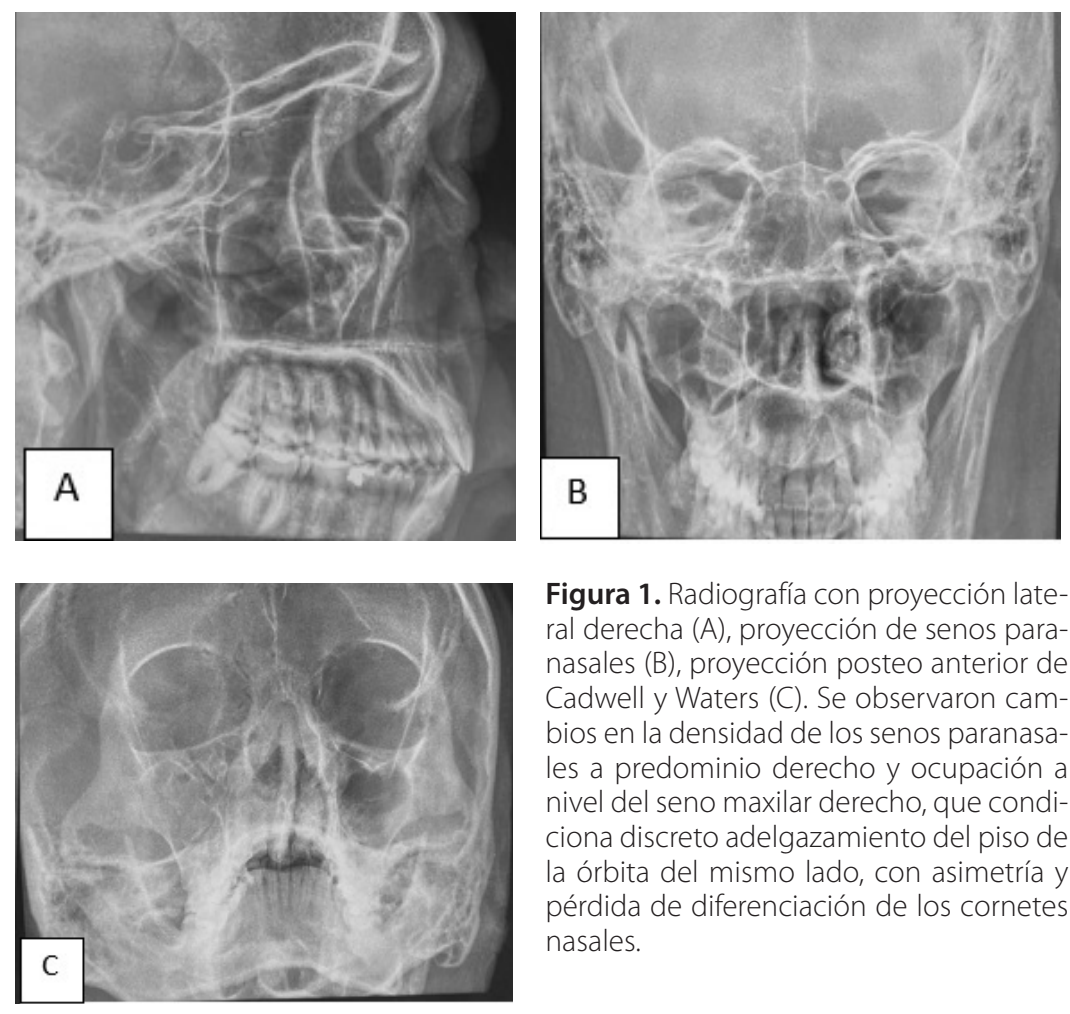

Figura 1. Radiografía con proyección lateral derecha $(A)$, proyección de senos paranasales (B), proyección posteo anterior de Cadwell y Waters (C). Se observaron cambios en la densidad de los senos paranasales a predominio derecho y ocupación a nivel del seno maxilar derecho, que condiciona discreto adelgazamiento del piso de la órbita del mismo lado, con asimetría y pérdida de diferenciación de los cornetes nasales.
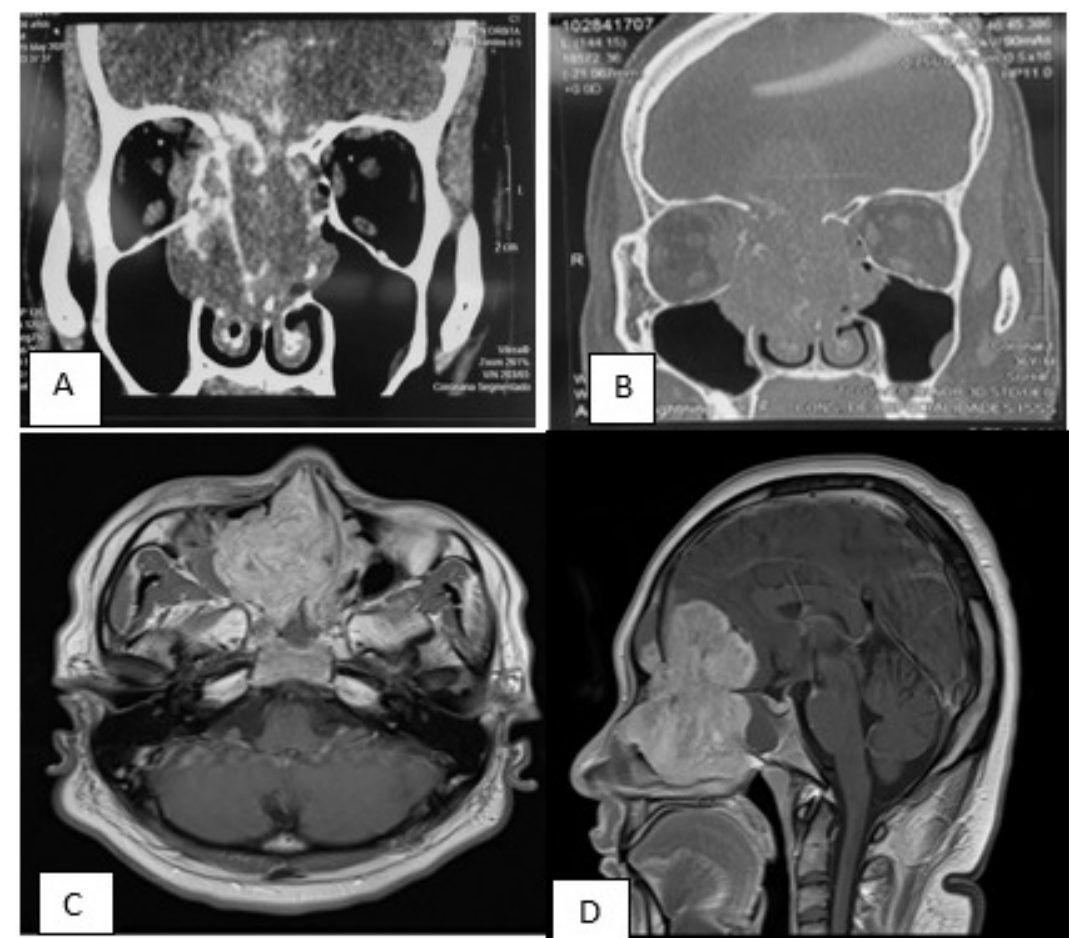

Figura 2. Tomografía de senos paranasales en corte coronal (A) con contraste y (B) sin contraste. Se observó una masa de tejidos blandos con infiltración, destrucción y extensión a ambos senos maxilares, paredes mediales de las órbitas y desplazamiento al músculo recto medial derecho lateralmente. Resonancia magnética cerebral (C) T1 corte axial y (D) corte sagital con contraste ferromagnético (gadolinio), que mostró un realce ávido de la lesión, de características infiltrantes a lámina cribiforme y hueso frontal con extensión a región intraconal derecha. No tenía extensión local a ganglios cervicales o torácicos (dato no evaluable en cortes presentados). 


\section{Intervención terapéutica}

El equipo multidisciplinario decidió la intervención quirúrgica temprana, a través del abordaje endoscópico híbrido nasal combinado con exéresis anterior frontal. Los hallazgos transquirúrgicos se delimitaron a la tumoración con compromiso de tejidos blandos, extensión intracraneal y se logró la exéresis completa de la lesión que abarcaba ambos senos maxilares, piso de órbita derecha e infiltración a región intraconal derecha con su respectivo nervio óptico y se decidió la enucleación del globo ocular derecho. El tiempo quirúrgico del proce-dimiento fue de siete horas. El paciente requirió ventilación mecánica invasiva y traslado a la unidad de cuidados intensivos.

Se envió estudio histopatológico de muestras de 0,3 y 0,7 cm, que reportaron la presencia de células monomórficas de mediano tamaño, con núcleos hipercromáticos, pleomorfismo moderado, citoplasma amplio aclarados y algunos eosinofílicos, con formación focal de rosetas de HomerWright (Figura 3).

\section{Evolución clínica}

El paciente recibió cuidados neurocríticos posteriores a la intervención, ventilación mecánica con traqueostomía y soporte aminérgico. El paciente falleció durante estancia hospitalaria en cuidados críticos debido a la severidad y extensión de la lesión. Según los parámetros de severidad y extensión intracraneal, los tumores considerados tipo Kadish C, con extensión intracraneal, poseen un porcentaje de mortalidad cercano al 92 $\%$ en el trans y posoperatorio, aun cuando fuere exitosa la exéresis completa de la lesión ${ }^{17}$.

\section{Diagnóstico clínico}

De acuerdo con los hallazgos encontrados en inmunohistoquímica en tinciones de cromogranina, sinaptofisina y del marcador CD56, se llegó al diagnóstico de neuroblastoma olfatorio de la región nasal derecha.

\section{Discusión}

El neuroblastoma olfatorio, por lo general, presenta síntomas inespecíficos, esto dificulta su diagnóstico precoz?. La media de retraso entre el primer síntoma y el diagnóstico es de seis meses. En el $70 \%$ de los casos aparece obstrucción nasal bilateral, epistaxis, hiposmia y cefalea, síntomas comunes en otras patologías, como la enfermedad sinusal polipoidea alérgica o la rinosinusitis crónica?

Los pacientes a menudo consultan en estadios avanzados con anosmia y alteraciones visuales', destrucción de las estructuras vecinas, como la órbita, el cerebro, tejidos blandos faciales y la piel, debido a tumores grandes, que pueden extenderse al compartimento intracraneal ${ }^{1}$. Esta invasión puede ser superior en la fosa craneal anterior, lateralmente en las órbitas y a través de la línea media en la cavidad nasal contralateral. También pueden obstruir la unidad osteomeatal de los senos paranasales, esto ocasiona opacificación del seno con secreciones ${ }^{1}$. Localmente, la enfermedad avanzada puede presentar signos de invasión 6 .

La importancia del diagnóstico precoz por imagen y terapéutico permite un manejo multidisciplinario en futuros pacientes ${ }^{8}$.

A través de una rinoscopía ${ }^{7}$ se puede observar una masa no distinguible de otras afecciones, como la poliposis, la sinusitis crónica u otras neoplasias malignas de la cavidad nasal ${ }^{8}$.
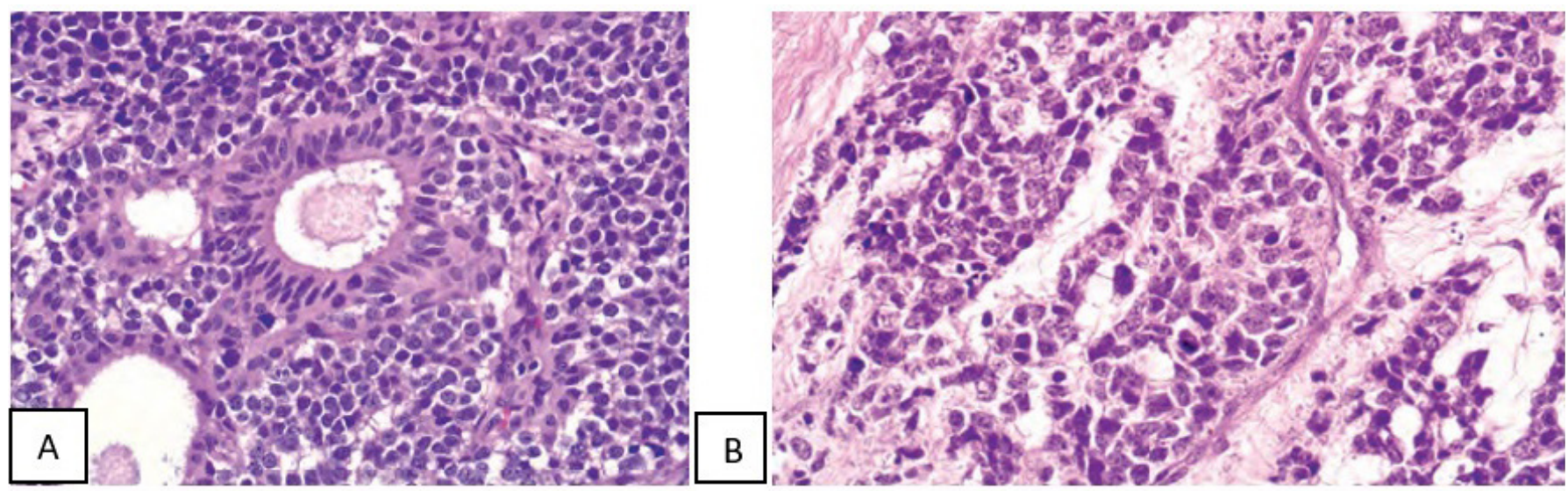

Figura 3. Cortes microscópicos a 40× (A, B), muestra del paciente anterior posexéresis quirúrgica en donde hay presencia de pleomorfismo nuclear de moderado a marcado con presencia de 20 mitosis por 10 campos de alto poder. No hubo evidencia de necrosis en lo examinado. Estos hallazgos son compatibles con neuroblastoma olfatorio. 
La tomografía axial computarizada y la resonancia magnética son importantes para identificar la extensión, el estadio del tumor y el abordaje quirúrgico'.

Para el manejo de esta enfermedad se han empleado múltiples estrategias, desde cirugía exclusiva, asociaciones de radioterapia y quimioterapia, cirugía y radioterapia hasta la asociación de las tres armas terapéuticas: cirugía, radioterapia y quimioterapia-14.

La supervivencia a los cinco años es del $70 \%$ aproximadamente ${ }^{15}$. La estrategia terapéutica que parece conseguir menor índice de recurrencia local $(10 \%)^{16}$, es la cirugía con resección craneofacial, seguida de radioterapia externa, siendo posible, tras la recurrencia local, el rescate terapéutico en el 33-50 \% ${ }^{1,17}$ de los casos.

\section{Aspectos éticos}

El caso presentado refleja información obtenida de los registros clínicos. No se realizó ninguna intervención o modificación intencionada de los datos del paciente. Se respetó la confidencialidad del paciente y los datos en la publicación han sido utilizados para fines académicos.

\section{Agradecimiento}

Al Departamento de Radiología e Imágenes del Hospital General del Instituto Salvadoreño del Seguro Social por su aporte tecnológico para la realización del estudio. A los departamentos de Patología, Otorrinolaringología y Neurocirugía por su apoyo invaluable en el abordaje terapéutico, quirúrgico y diagnóstico de esta compleja entidad.

\section{Financiamiento}

Autores declaran no tener fuente de financiamiento.

\section{Referencias bibliográficas}

1. Navas-Campo R, Moreno Caballero L, Gasos Lafuente A, Tobajas Morlana P, Séez Valero E, Gimeno Peribáñez MJ. Neuroblastoma olfatorio. Todo lo que el radiólogo debe saber. Rev Argent Radiol /Argent J Radiol. 2020;84(01):017-29. DOI: 10.1055/s-0040$\underline{1702993}$

2. Benet MS, Garabal GJA, Luis HJ, Tacoronte PL, Artazkoz TJJ. Estesioneuroblastoma o neuroblastoma olfatorio. Rvdo. Otorrinolaringología. Cir. Head Neck. 2017;77(1):63-68. DOI: 10.4067/S071848162017000100009
3. Chavarría Elizondo D, Carranza Rojas R. Estesioneuroblastoma: reporte de caso y revisión bibliográfica. Rev. Costarric. Salud Pública. 2019;28(1):83-95. Disponible en: http://www.scielo.sa.cr/scielo. php? script=sci arttext\&pid $=$ S1409$14292019000100083 \& \operatorname{lng}=e n$

4. Su SY, Bell D, Hanna EY.

Esthesioneuroblastoma, neuroendocrine carcinoma, and sinonasal undifferentiated carcinoma: differentiation in diagnosis and treatment. Int Arch Otorhinolaryngol. 2014;18(2):149-56. DOI: 10.1055/s-00341390014

5. Soldatova L, Campbell RG, Carrau RL, Prevedello DM, Wakely P Jr, Otto BA, et al. Sinonasal carcinomas with neuroendocrine features: Histopathological differentiation and treatment outcomes. J Neurol Surg B Skull Base. 2016;77(6):456-65. DOI: $10.1055 /$ s-0036-1582432

6. Menon S, Pai P, Sengar M, Aggarwal JP, Kane SV. Sinonasal malignancies with neuroendocrine differentiation: case series and review of literature. Indian J Pathol Microbiol. 2010;53(1):28-34. DOI: $10.4103 / 0377-4929.59179$

7. Anchia-Guerra N, López-Rodríguez I, Herrera-Wainshtok A, Wainshtok-Tomás D, Enamorado-Suárez E, Vargas-Peña M. Estesioneuroblastoma. Revista Cubana de Otorrinolaringología y Cirugía de Cabeza y Cuello. 2021;5(3) Disponible en: http://www. revotorrino.sld.cu/index.php/otl/article/ view/185

8. Mehta GU, Raza SM, Su SY, Hanna EY, DeMonte F. Management of olfactory neuroblastoma, neuroendocrine carcinoma, and sinonasal undifferentiated carcinoma involving the skullbase. J Neurooncol. 2020;150(3):367-75. DOI: $10.1007 /$ s11060020-03537-1

9. Bell D, Hanna EY, Weber RS, DeMonte F, Triantafyllou A, Lewis JS Jr., et al. Neuroendocrine neoplasms of the sinonasal region. Head Neck. 2016;38 (1):E2259-66. DOI: $10.1002 /$ hed.24152

10. Bell D. Sinonasal neuroendocrine neoplasms: Current challenges and advances in diagnosis and treatment, with a focus on olfactory neuroblastoma. Head Neck Pathol. 2018;12(1):22-30. DOI: $10.1007 /$ s12105-018-0887-5

11. Shah K, Perez-Ordóñez B. Neuroendocrine neoplasms of the sinonasal tract: Neuroendocrine carcinomas and olfactory neuroblastoma. Head Neck Pathol. 2016;10(1):85-94. DOI: 10.1007/s12105-016$\underline{0696-7}$

12. Turri-Zanoni M, Maragliano R, Battaglia $P$, Giovannardi M, Antognoni P, Lombardi D, et al. The clinicopathological spectrum of olfactory neuroblastoma and sinonasal 
neuroendocrine neoplasms: Refinements in diagnostic criteria and impact of multimodal treatments on survival. Oral Oncol. 2017;74:21-9. DOI: 10.1016/j. oraloncology.2017.09.010

13. Xiong L, Zeng X-L, Guo C-K, Liu A-W, Huang L. Optimal treatment and prognostic factors for esthesioneuroblastoma: retrospective analysis of 187 Chinese patients. BMC Cancer. 2017;17(1):254. DOI: 10.1186/ s12885-017-3247-z

14. Sun M, Wang K, Qu Y, Zhang J, Zhang $\mathrm{S}$, Chen $\mathrm{X}$, et al. Long-term analysis of multimodality treatment outcomes and prognosis of esthesioneuroblastomas: a single center results of 138 patients. Radiat Oncol. 2020;15(1):219. DOI: 10.1186/s13014$\underline{020-01667-4}$
15. Li R, Tian S, Zhu Y, Yan L, Zhu W, Quan H, et al. Management of orbital invasion in esthesioneuroblastoma: 14 years' experience. Radiat Oncol. 2019;14(1):107. DOI: $\underline{10.1186 / \text { s13014-019-1313-1 }}$

16. Dulguerov P, Allal AS, Calcaterra TC. Esthesioneuroblastoma: a meta-analysis and review. Lancet Oncol. 2001;2(11):683-90. DOI: $10.1016 /$ S1470-2045(01)00558-7

17. Rojo Sanchis N, Cambra Marti MR, Marin Canete A, Barragan Requena BY, Vera Llaó R, Sanchez Laforga AM. Evaluación radiológica de los tumores nasosinusales. 2014;56. DOI: $\underline{10.1594 / \text { seram2014/S-0887 }}$ 